\title{
Development and Innervation of Soleplates in the Freely Grafted Extensor Digitorum Longus (EDL) Muscle in the Rat
}

\author{
FAY M. HANSEN-SMITH \\ Department of Anatomy, The University of Michigan Medical School, Ann. \\ Arbor, MI 48109 and Department of A natomy, The University of Detroit \\ School of Dentistry, Detroit, MI 48207
}

\begin{abstract}
The ultrastructural events in the establishment of the neuromuscular junction of the freely grafted extensor digitorum longus (EDL) muscle of the rat were studied 1-120 days after grafting. The original axons and muscle fibers, including soleplates, degenerated during the first few days, but Schwann cells and basal laminae persisted. Myofibers regenerated within the original basal laminae. Indentations of the sarcolemma, termed "presumptive synaptic clefts" (PSC), were found on myotubes from 7-day grafts. Schwann cells and residual acetylcholinesterase were invariably associated with the PSC, suggesting that the PSC developed at the site of the original soleplate. Nerves entered the grafts 10 days postoperatively and contacted the PSC of the regenerating muscle fibers on the 18-20th day. The secondary synaptic clefts of these "reconstructed" soleplates extended far beyond the subaxonal region. A second type of soleplate appeared on the 18-20th day. These soleplates were similar to those found in embryonic muscle and were considered to have been induced to form "de novo" by the presence of the nerves. When grafts were placed in permanently denervated limbs the "reconstructed" soleplates appeared, but the "de novo" type did not. These results show that information directing the morphogenesis and innervation of the soleplate persists after the original muscle fibers and axons of a graft degenerate and regenerate.
\end{abstract}

It has been known for some time that regeneration of skeletal muscle fibers is possible after the original fibers have been injured (Clark et al., 1947; Albrook and Aitken, 1951; Studitsky et al., 1963; Carlson, 1973, 1978; Carlson et al., 1979a). One model that has been used extensively for the study of muscle regeneration is the freely grafted extensor digitorum longus (EDL) muscle of the rat. In this model the EDL is removed and then replaced into its original bed without neural or vascular anastamoses. New muscle fibers regenerate following complete destruction of the original muscle fibers within the grafted EDL. During the first few days the original muscle fibers undergo an intrinsic destruction as a result of the ischemia. As the grafts become revascularized the necrotic debris of the muscle fibers is phagocytized, leaving basal laminae that contain presumptive myoblasts. Between the fourth and seventh day after grafting the presumptive myo- blasts proliferate, and myoblasts and myotubes are formed within the persisting original basal laminae. The regenerating myotubes and immature myofibers become covered by some new, closely adherent basal laminae by the 18-20th day, and redundant original basal lamina is degraded (HansenSmith and Carlson, 1979).

Nerves are first observed in rat EDL grafts 10 days after operation, but innervation of the regenerated fibers does not occur until the end of the third week (Carlson et al., 1979b). In this study the freely grafted EDL muscle was used to examine the chronological events in the establishment of the neuromuscular junction in regenerating mammalian muscle. The objective was to describe the morphogenesis of the soleplate region and to observe the relationship of these soleplates to the axons innervating the regenerating

\footnotetext{
Received August 23, 1982; accepted April 27, 1983.
} 
fibers. The results show that the morphogenesis of the soleplate precedes innervation and may occur in the complete absence of innervation. The "reconstruction" of soleplates thus differs from de novo soleplate formation. However, there is also evidence suggesting that a nerve-dependent de novo formation of soleplates may occur in some regions of a graft. The morphology of the soleplates in mature, regenerated mammalian muscle fibers differs in some respects from that in control, nonregenerating muscle.

\section{METHODS}

The EDL muscles from 52 male SpragueDawley rats (175-200 gm) were freely grafted while the rats were anesthetized by ether. As previously described (Carlson and Gutmann, 1976), the muscles were teased away from their tendonous attachments and were severed from their neurovascular supply. The excised muscles were injected with Marcaine $(0.7 \%$, Winthrop) and immersed in the Marcaine solution for 10 minutes to destroy all of the original muscle fibers (Carlson and Gutmann, 1976). The muscles were then sutured to the tendons of origin and insertion in the original bed, without neural or vascular anastomoses. Contralateral muscles were used as controls. In a second series of experiments (six rats), the hind limbs on both experimental and control sides were permanently denervated by removing approximately $1 \mathrm{~cm}$ of the sciatic nerve in the thigh and suturing the proximal stump to the skin at the time of the grafting operation.

The grafts and control muscles were removed after $1,4,7,10,15,18,20,23,30,35$, 43,60 , or 120 days. Muscles from rats with denervated limbs were removed $7,15,20,30$, 43 , or 60 days after grafting. Sample blocks for electron microscopy were taken from the proximal half of the muscles and fixed overnight in $2 \% 0.2 \mathrm{M}$ cacodylate-buffered glutaraldehyde $(\mathrm{pH} 7.38)$ at $4^{\circ} \mathrm{C}$. All of the early grafts were fixed by immersion. Approximately half of the maturing and mature grafts (23-120 days) were fixed by perfusion via the abdominal aorta. The tissues for electron microscopy were postfixed in $1 \% 0.2 \mathrm{M}$ cacodylate-buffered osmium tetroxide and embedded in Spurr's resin. Survey sections $(1 \mu \mathrm{m})$ were stained with toluidine blue. Pale gold sections were placed on 200-mesh copper grids, stained with uranyl acetate and lead citrate and were examined with a Hitachi HU-11A microscope.
To aid in the localization of soleplates by electron microscopy, sample blocks in which survey sections showed the presence of intact or degenerated nerves were sectioned at one or more levels and examined for the presence of soleplates. In addition, the distribution of soleplates in whole muscles or in muscle fiber bundles was traced by reacting the fixed specimens for acetylcholinesterase (Karnovsky, 1964; Goshgarian, 1977). Incubation times varied with the specimen. Prolonged incubations were frequently necessary to visualize the reaction product, particularly in 7-20-day grafts. The reacted specimens were examined under a dissecting microscope and reactive regions were isolated from the grafts. These specimens were subsequently embedded, sectioned, and examined by electron microscopy. It is emphasized that for this study the technique was used solely to determine the general location of acetylcholinesterase activity in order to improve the chances of finding soleplates in the samples. The exact ultrastructural location of the enzyme was not considered reliable because of the possibility of diffusion of the reaction product during prolonged incubations.

\section{RESULTS \\ Terminology}

For this report the term "soleplate" refers to the specialized region of the plasmalemma and the sarcoplasm that underlies the axon at the neuromuscular junction of intact control muscle. Two types of surface specializations invaginate into the soleplate region of control muscle fibers. Primary synaptic clefts are shallow depressions in which the axon lies. Numerous deep secondary synaptic clefts, formed by the invagination of sarcolemma and basal laminae, extend into the sarcoplasm from the primary synaptic clefts. In the present report the term "soleplate" is also used to refer to a comparable area of the muscle fiber in denervated or regenerating muscle where the secondary "synaptic" clefts, but not necessarily the primary clefts, are present.

\section{Controls}

The structure of soleplates in the nongrafted control EDL muscle is identical to that described by other investigators (Fig. 1). Secondary synaptic clefts are relatively deep, typical of those described for the EDL and other "fast" or "white" muscles (Ogata and Murata, 1969; Padykula and Gauthier, 1970). 

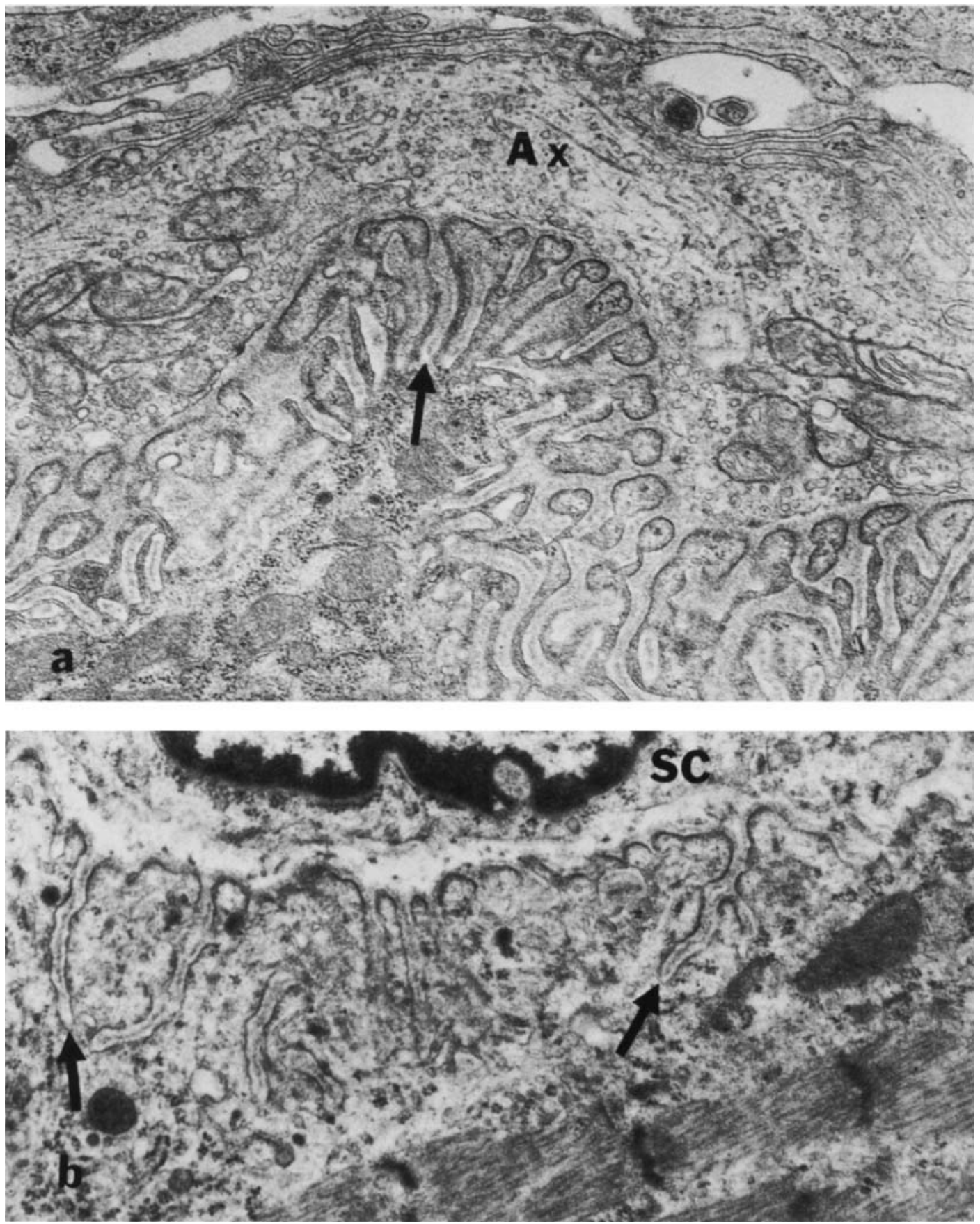

Fig. 1. a) Soleplate from a control EDL. $\times 21,000$. b) Soleplate from a non-grafted EDL 14 days following de- nervation. Arrows indicate secondary synaptic clefts. $\times 15,500$. Schwann cell (SC), axon (Ax). 


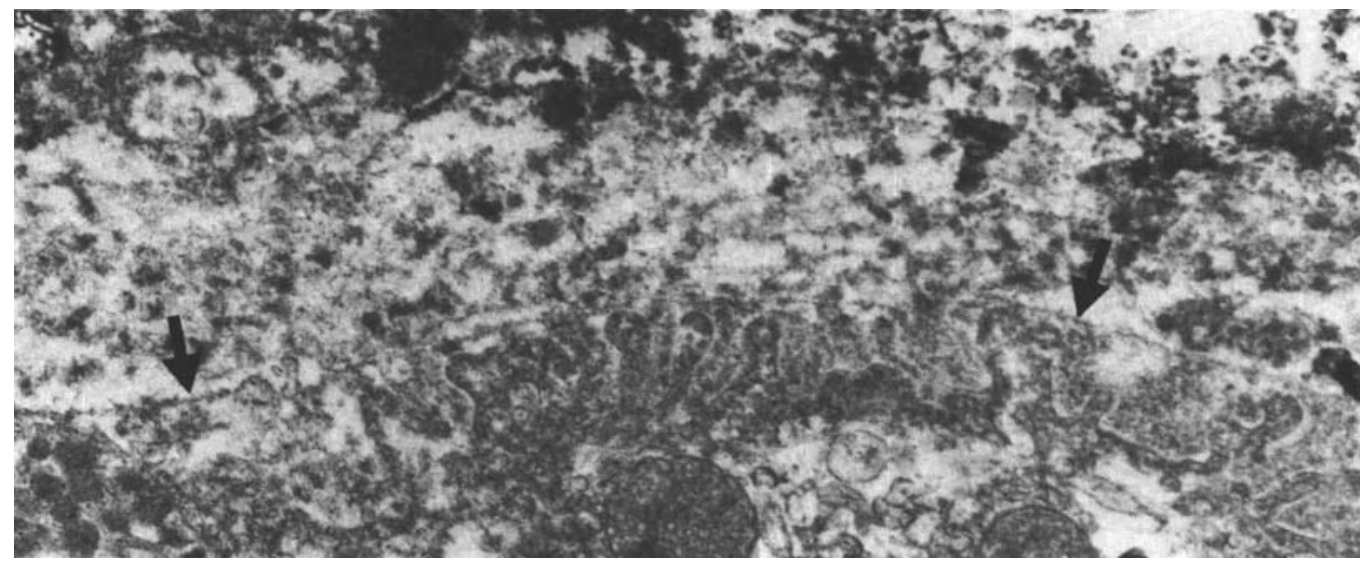

Fig. 2. Electron-dense acetylcholinesterase reaction with secondary synaptic clefts have degenerated, but product in the ischemic region of a 4 -day graft. Basal clumps of remaining sarcoplasm mark the original synlamina (arrows) persists. Plasma membranes associated aptic region. $\times 20,500$.

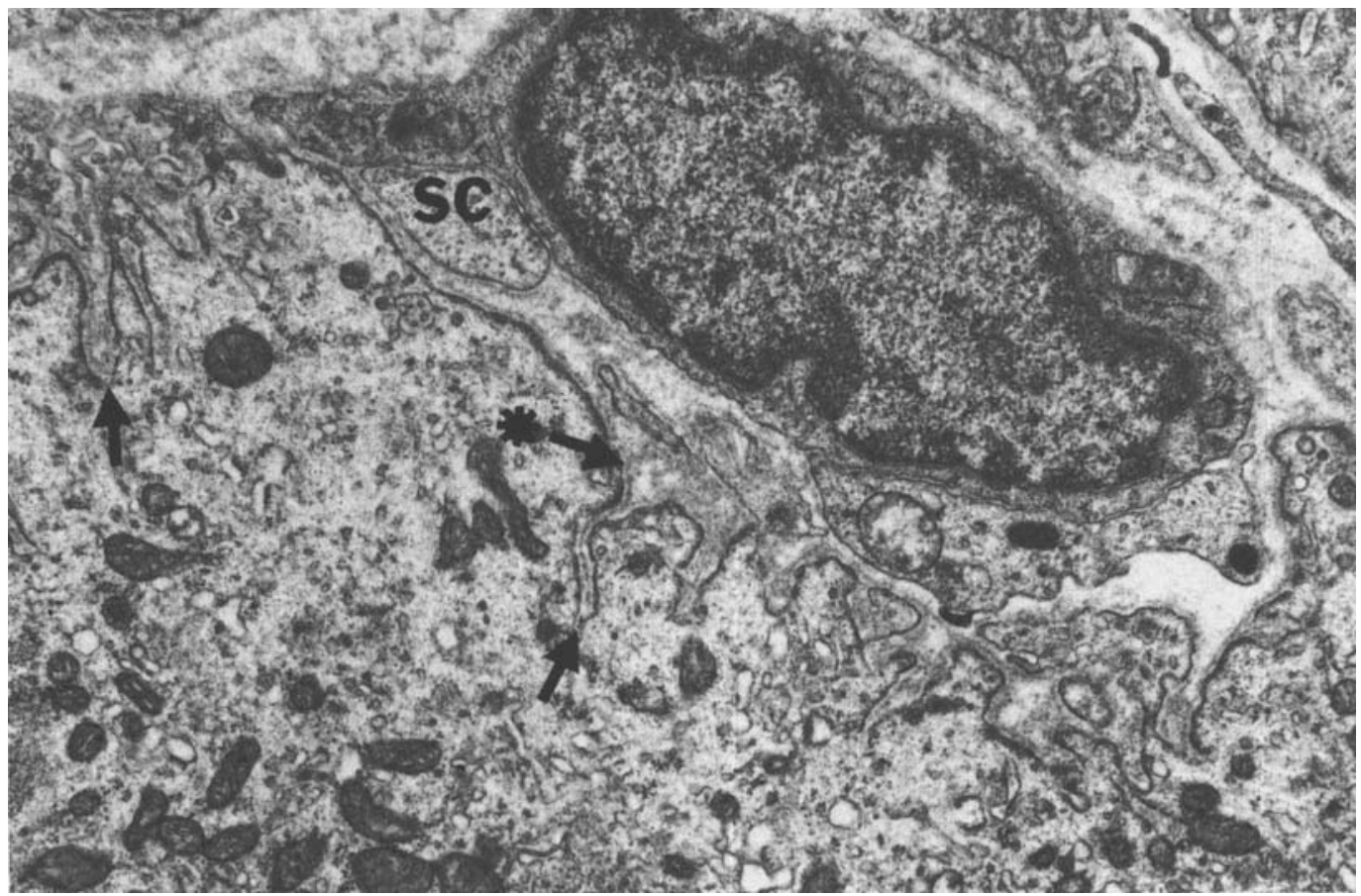

Fig. 3. Presumptive synaptic clefts (arrows) in myotube from a 7-day graft. Schwann cells (SC) are found in close proximity. Redundant basal laminae are present ("arrow). $\times 23,000$ 


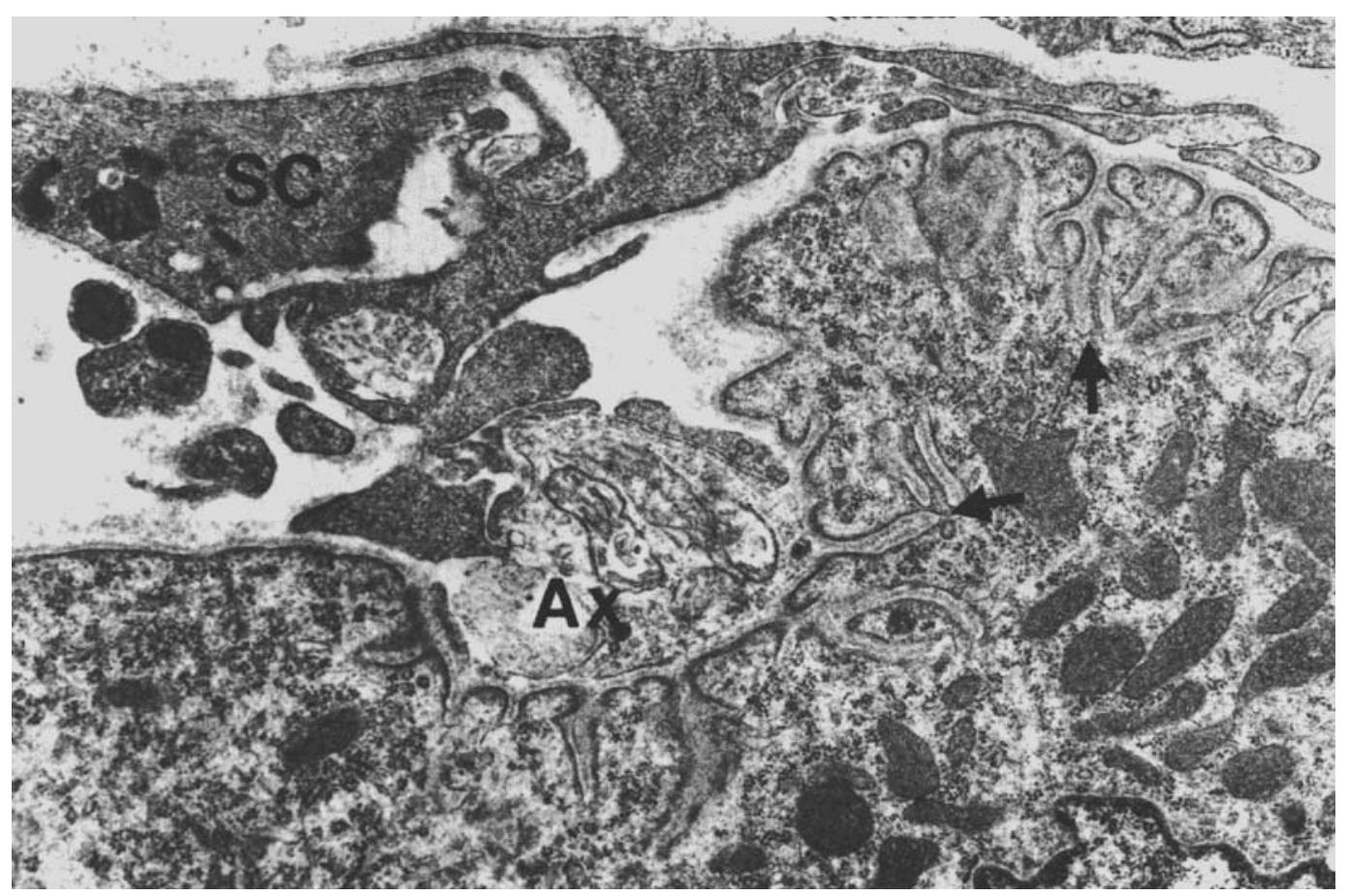

Fig. 4. Neuromuscular junction in a 20-day graft. The region of secondary "synaptic" clefts (arrows) extends

For the purpose of this report it is emphasized that the region of secondary synaptic clefts was rarely found to extend beyond the area of the soleplate immediately beneath the axon. In denervated muscles the primary synaptic clefts were shallower or absent. The secondary synaptic clefts were not markedly changed although some appeared to be more irregular in size (Fig. 1).

\section{Muscle Degeneration}

Degenerating muscle fibers are found in the grafts 1-4 days after operation. Only a few traces of the original soleplates are evident in the degenerating muscle fibers (Fig. 2 ). The overlying basal lamina persists. The specific indentation pattern characteristic of the secondary clefts is not usually maintained. Although axons degenerate, the surrounding Schwann cells and perineuronal cells survive, but usually retract from the original synaptic area, leaving "empty" basal laminae. beyond the subaxonal region. Schwann cells (SC), axon $(\mathrm{Ax}) . \times 18,000$.

\section{Muscle Regeneration Prior to Innervation (4-18-Day Grafts)}

Sarcolemmal infoldings oriented perpendicularly to the surface of the myotubes are found as early as 7-10 days after grafting (Fig. 3). For the purpose of this report these structures will be referred to as "presumptive secondary clefts" (PSCs) because of their resemblance to the secondary synaptic clefts associated with the soleplates of nonregenerating muscle. When the PSCs first appear there are only a few infoldings, usually irreg. ularly spaced, but by $15-18$ days a complex and extensive system of invaginations has developed. The organization resembles those of the secondary clefts in the soleplates of denervated, nonregenerating muscle. The PSCs may be covered by either a single or double layer of basal lamina. The plasmalemma at the surface of the PSCs is thickened and electron-dense relative to that in the non-PSC region of the same myotubes and regenerating muscle fibers. There is no 

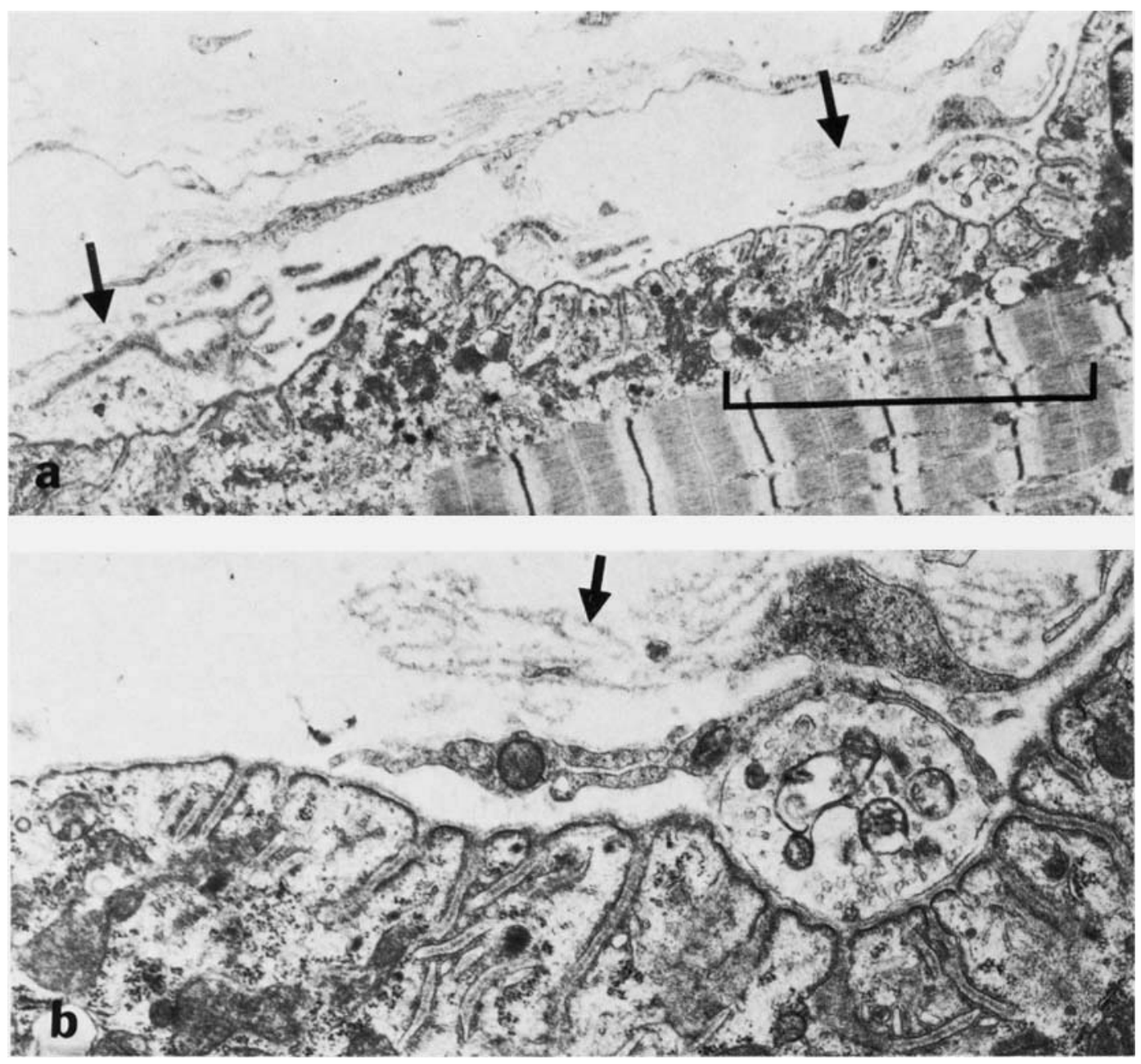

Fig. 5, a) Soleplate from a 35-day graft. Region of secondary synaptic clefts extends laterally beyond the sub-axonal region. Arrows indicate redundant basal laminae associated with Schwann cells. $\times 11,400$. b) Region around axon on right side of above figure is enlarged $\times 25,000$. preferential aggregation of myonuclei associated with the PSCs in the early grafts, but one or more myonuclei are commonly associated with these sites as regeneration progresses. Axonal debris and/or Schwann cells from the original nerve are invariably associated with the PSCs-i.e., if not immediately adjacent to the PSCs, the sheaths of degenerated neurons and Schwann cells are at least within the field of view on the same or an adjacent grid square. The Schwann cells often have enlarged, euchromatic nuclei. Loose, redundant basal laminae from the original muscle fibers and nerves are fre- quently associated with both the PSC and the non-PSC region of both the myotubes and Schwann cells (Fig. 3). Residual acetylcholinesterase is detected in the interstitium overlying the PSCs.

\section{Innervation of Regenerated Muscle Fibers (18 Days or Later)}

The first actual contacts of axons with muscle fibers are detected 18-20 days postoperatively. The majority of contacts are at highly specialized sites identical to the PSCs that were observed a few days previously. The secondary clefts associated with the soleplate 

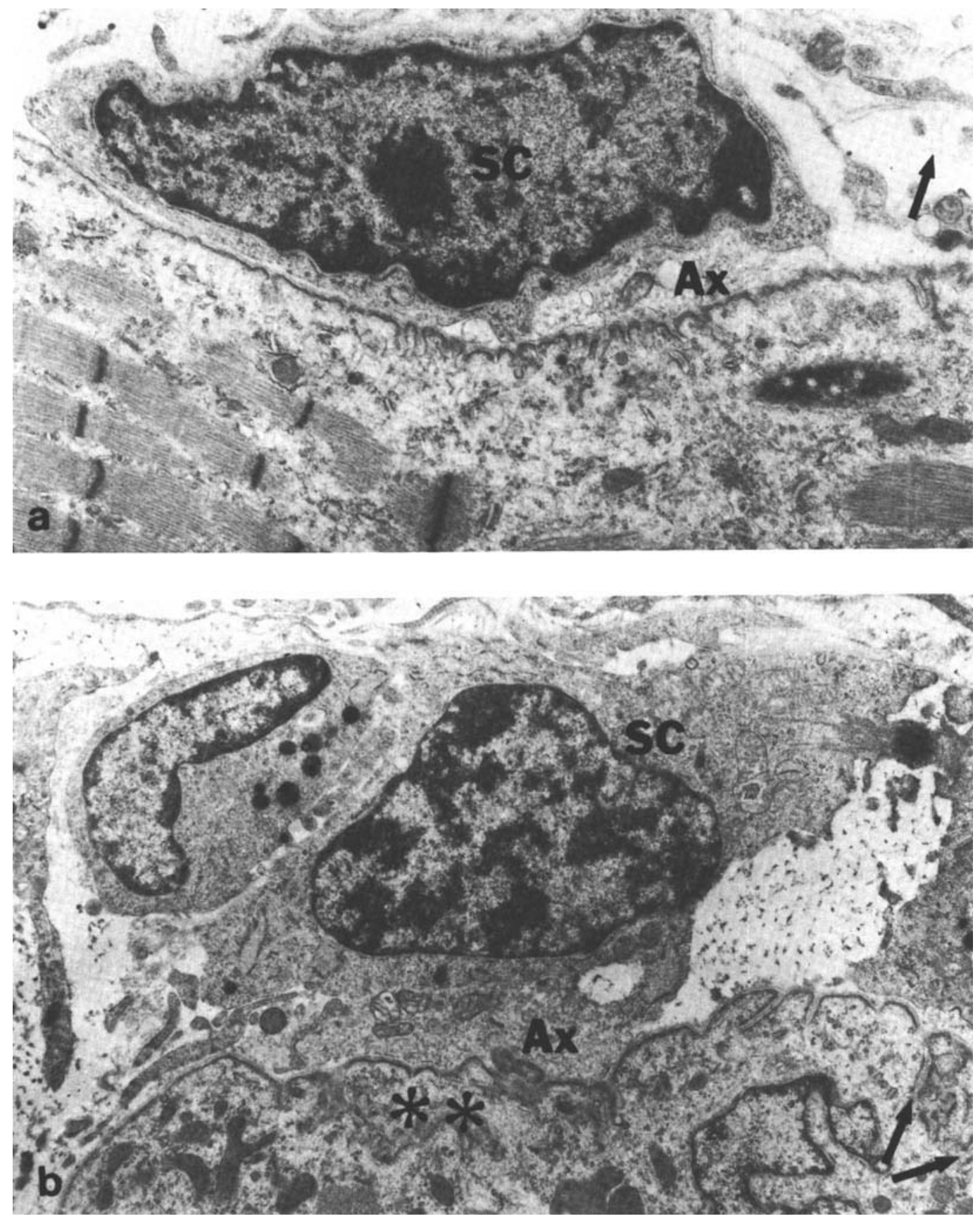

Fig. 6. a) Soleplate from a 30-day graft consists of shallow pits that are restricted to the region immediately beneath the axon. Arrows indicate loose basal laminae overlying the region. $\times 12,000$. b) Soleplate from a 20-day graft contains both deep synaptic clefts (arrows) and shallow pits (asterisks). Schwann cell is undergoing mitosis. $\times 12,000$. Schwann cell (SC), axon $(\mathrm{Ax})$. 


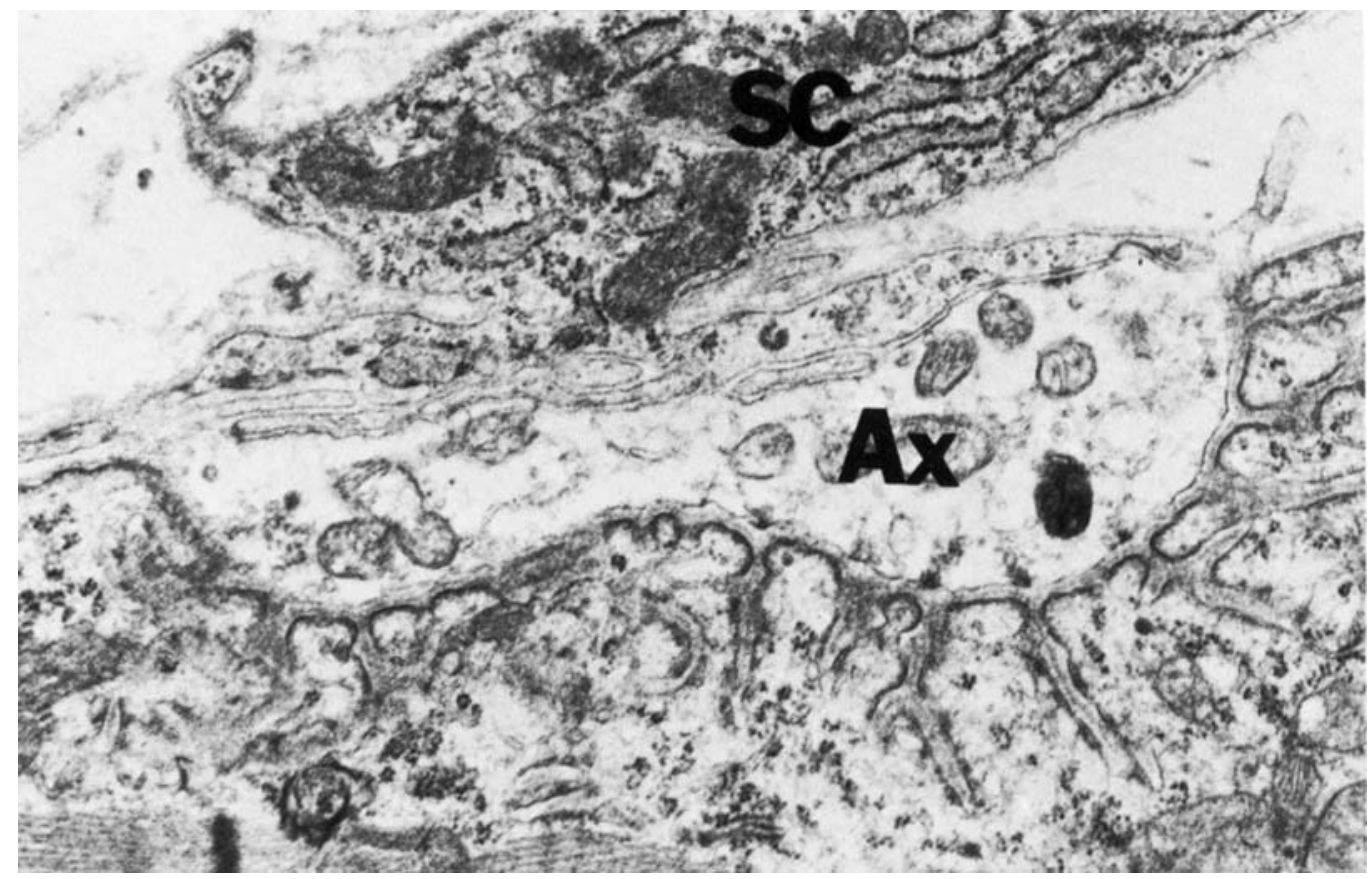

Fig. 7. Soleplate from a 43 day graft is similar to that of controls. Schwann cell (SC), axon (Ax). $\times 24,000$.

extend beyond the immediate subaxonal region (Fig. 4). At the time the axons contact the muscle fibers there are always Schwann cells closely associated with them. The Schwann cells normally enclose only one axon. Many of the Schwann cells have redundant basal laminae associated with them (Fig. 5), in addition to a closely adherent layer of basal lamina that fuses with that of the muscle fiber.

Four different types of soleplate structures are associated with the subaxonal regions of the regenerating muscle fibers onwards from the 20th day. The most common morphological configuration consists of regular, narrow, deep secondary clefts resembling those associated with soleplates in innervated, nonregenerating control muscle, except that the clefts usually extend for variable distances beyond the immediate subaxonal region (Fig. 5). A second type of specialization consists of shallow pits that are confined to the immediate subaxonal region (Fig. 6). The plasmalemma associated with both the deep secondary clefts and the shallow pits is thickened and more electron-dense than elsewhere on the muscle fiber. Both the shallow pits and deep secondary clefts are sometimes found within the soleplate region of the same muscle fiber (Fig. 6). Both types of soleplates are found on mature regenerated muscle fibers up to 120 days. A third type of soleplate is first observed in 30-35-day grafts. These are identical to control soleplates. Their regularly spaced secondary clefts are confined to the immediate subneuronal area (Fig. 7). In addition, noninnervated soleplates similar to those of nonregenerating denervated muscles are occasionally found. A summary of the various types of soleplates that may be found in mature grafts is diagrammed schematically in Figure 8.

\section{Grafts in Denervated Limbs}

PSCs are observed 7-10 days after grafting the EDL into a denervated hind limb. These structures are identical to those found in grafts in nondenervated limbs. As with the grafts in innervated limbs, the PSCs increase in complexity as regeneration progresses (Fig. 9).

\section{DISCUSSION}

The regeneration of skeletal muscle fibers mimics the developmental process in that undifferentiated stem cells proliferate and 


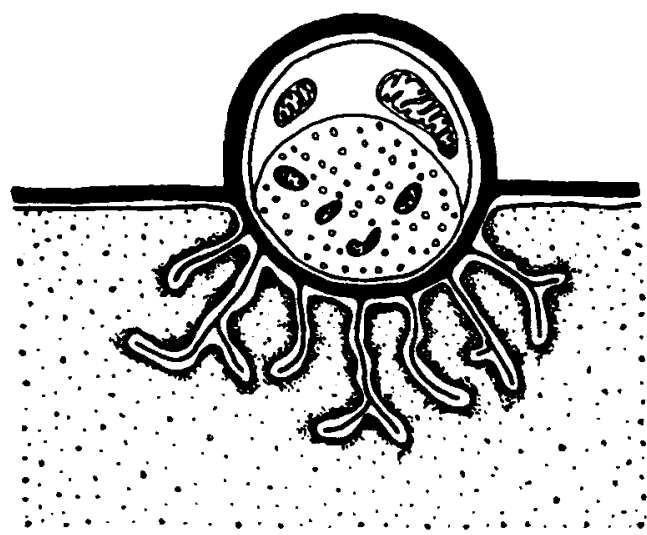

a

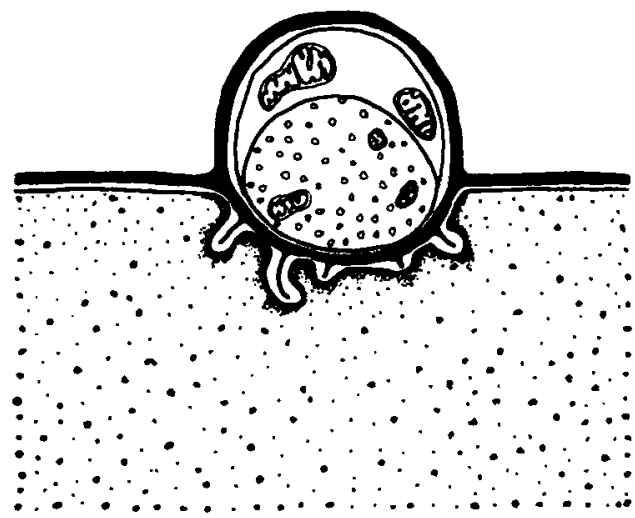

C

Fig. 8. Summary of types of soteplates found in mature EDL grafts. a) Soleplates resemble controls, i.e., secondary synaptic clefts are deep and are limited to subaxonal region. b) Secondary synaptic clefts are deep but extend beyond subaxonal region. c) Secondary syn-

differentiate sequentially into myoblasts, myotubes, and myofibers. Thus, it might be expected that the formation of soleplates in regenerating muscle fibers would also mimic the morphogenesis of soleplates at the neuromusclar junction during embryogenesis. The present chronological study suggests, however, that the morphogenesis of many of the soleplates in freely grafted EDL muscle does not proceed in the same way as in embryonic mammalian muscle (Hirano, 1967; Teräväinen, 1968; Kelly and Zacks, 1969;
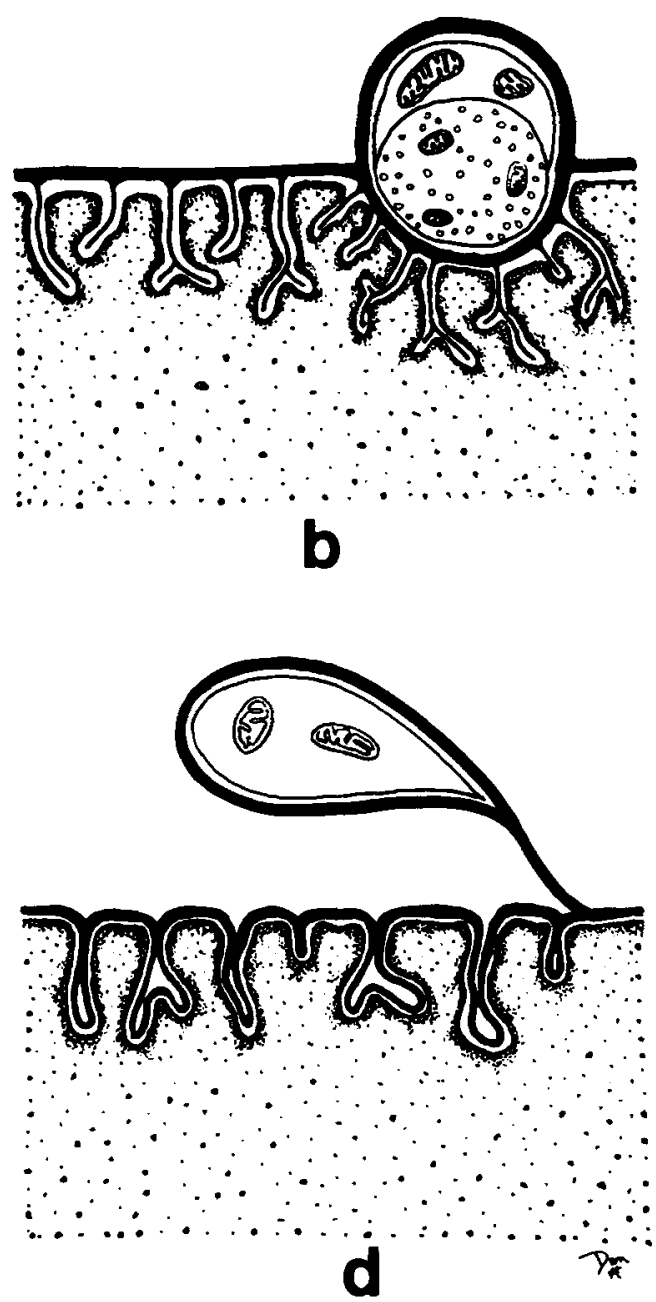

aptic clefts are shallow and are limited to subaxonal region. d) Secondary synaptic clefts are deep and irregular; axon and primary cleft are absent. Soleplate is not innervated.

Juntenen, 1971; Atsumi, 1977), muscle within a regenerating amphibian limb (Lentz, 1969), or ectopic soleplate formation in mature, nonregenerating muscle (Saito and Zacks, 1969; Koening, 1973; Kornielusson and Sommerschild, 1976). Whereas the preceding require the presence of an axon before the morphological specialization of the soleplate can develop, specializations are reconstructed in the regenerating EDL muscles as early as the myotube stage. The reconstruction of the soleplate does not require the pres- 


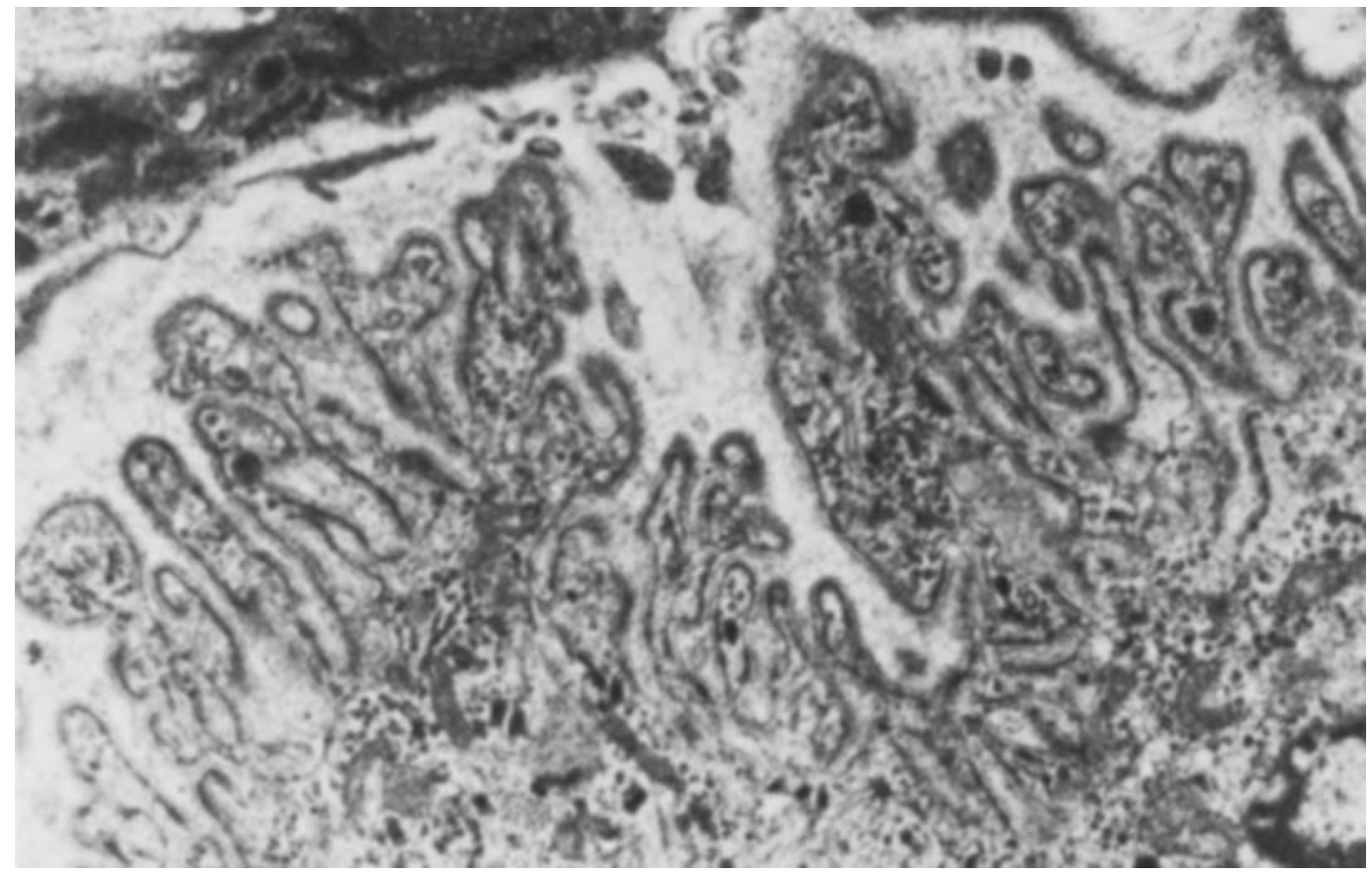

Fig. 9. Soleplate associated with an atrophic muscle fiber from a 30 day graft that was placed in permanently denervated limb. $\times 7,000$.

ence of an intact axon. A schematic summary of the morphological events in the nerve-independent mechanism of reconstruction of soleplates in regenerating muscle is shown in Figure 10.

Several lines of evidence suggest that the PSCs or the "reconstructed" soleplates in the regenerates probably developed at or near the sites of the soleplates in the original muscle. The PSCs were invariably close to the original, but degenerated axon sheaths. Schwann cells were frequently closely apposed to the PSCs and were usually at least partially surrounded by a loose basal lamina that also attached to the myotube at or near the PSC. Acetylcholinesterase activity was associated with the interstitium near the PSCs. Marshall et al. (1977) and Burden et al. (1979) have demonstrated that acetylcholinesterase is residual from the soleplate regions in the original muscle fibers, rather than being newly synthesized by the regenerating muscle fibers at this stage of regeneration. In addition, the soleplates in newly innervated, as well as mature regenerated muscle fibers, were often incompletely cov- ered by axons. The exposed secondary clefts at the onset of innervation strongly suggest that these clefts were present prior to innervation.

The PSCs found in the early regenerates, as well as the true synaptic clefts associated with soleplates in mature innervated grafts, were characterized by thickened electrondense membranes at the surface of the fibers. This morphological specialization of the sarcolemma corresponds to what is believed to be the site of acetylcholine receptors (Fertuck and Salpeter, 1974). Labeling studies with $\alpha$ bungarotoxin have demonstrated that acetylcholine receptors (AchRs) are found at the soleplates of mature regenerated mammalian or amphibian muscle. These receptors appear independently of innervation (Burden et al., 1979; Bader, 1981). Preliminary studies with $\alpha$-bungarotoxin suggest that AchR clusters first appear at about 7 days, when the PSCs appear (unpublished observations).

A second, less highly developed type of soleplate appeared only after axons had contacted the regenerated muscle fibers. These pitlike structures were similar to those 

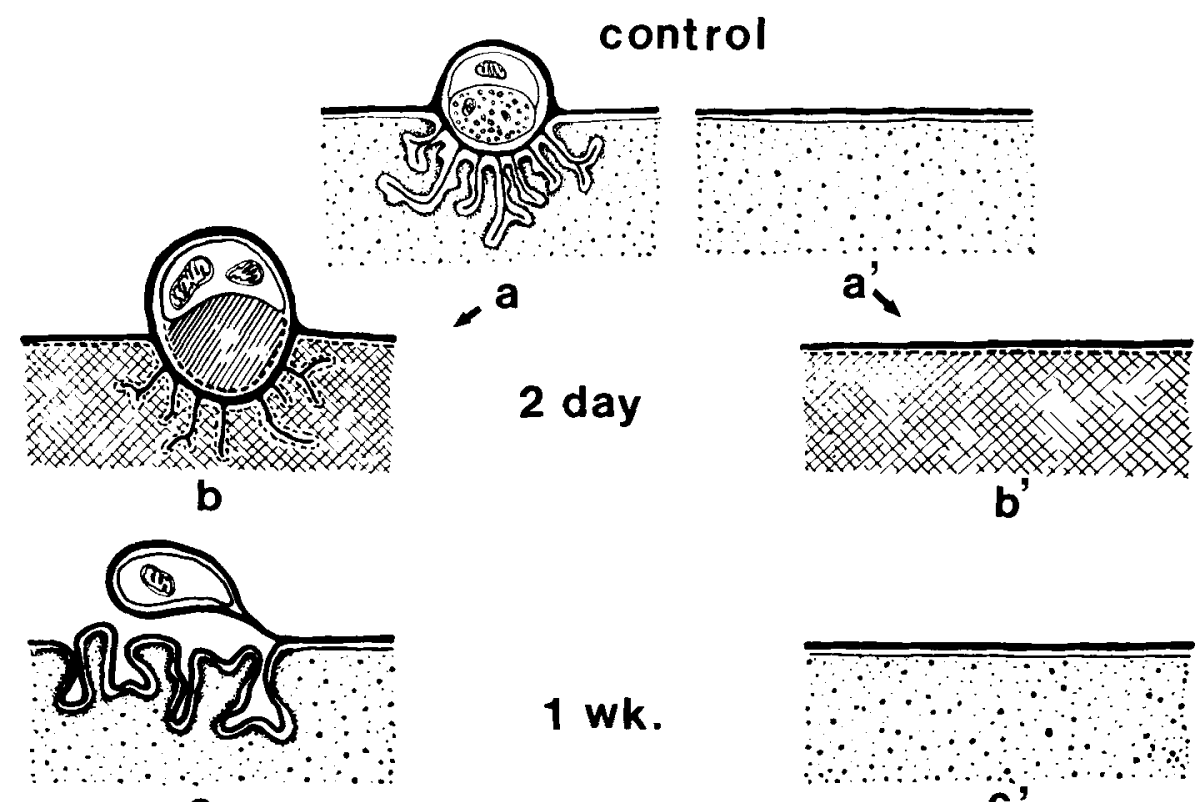

C

1 wk.
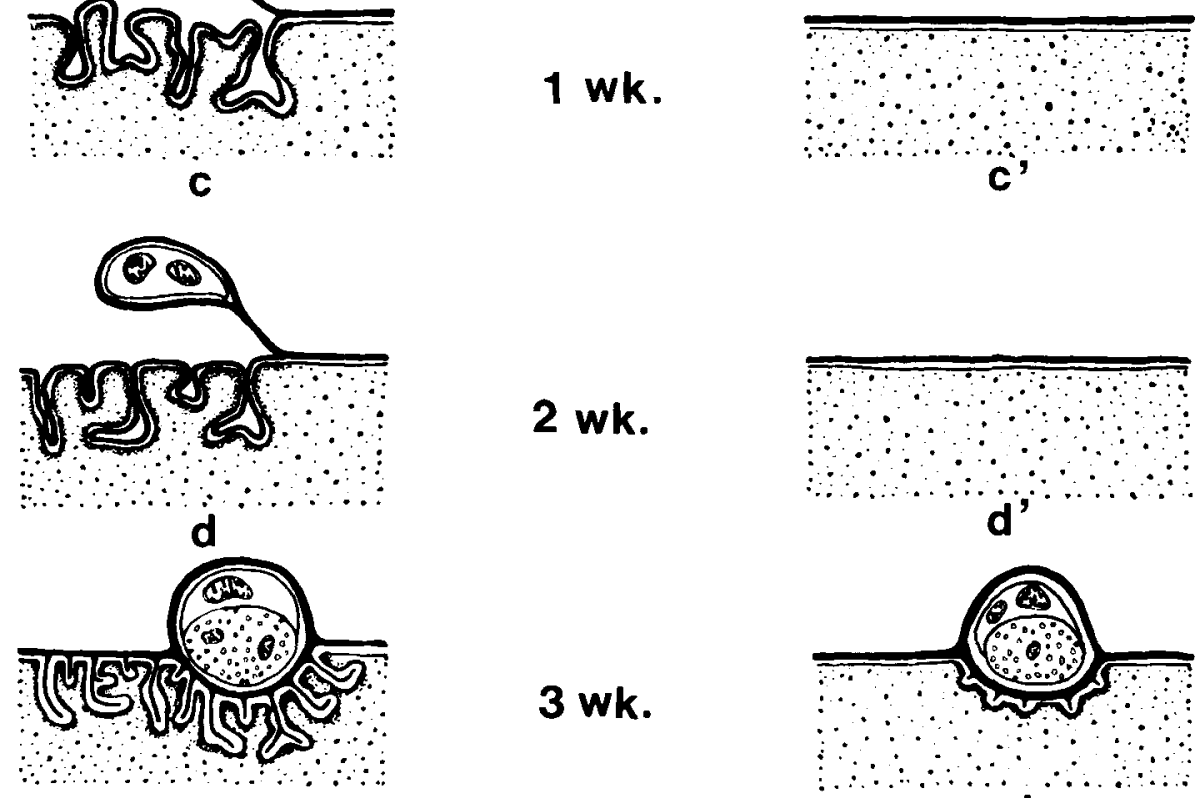

2 wk.

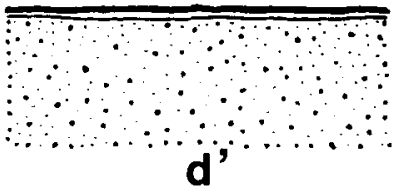

3 wk.

e

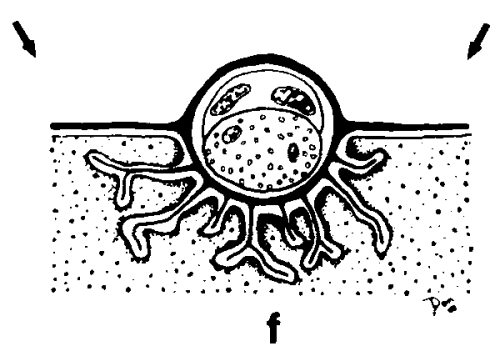

mature

Fig. 10. Schematic summary of soleplate morphogenesis in regenerating EDL. a and $a^{\prime}$ represent the neuromuscular junction (a) and extrajunctional region ( $a^{\prime}$ ) of an intact control muscle. b-e represent the hypothetical nerve-independent "reconstruction" sequence at 2 days, 1,2 , and 3 weeks: b) basal laminae and Schwann cells persist but axon and muscle fiber degenerate; ... ind i $_{\text {- }}$ cates absence of plasma membrane during degeneration. c) Irregular presumptive synaptic clefts form in the vicinity of the Schwann cell. d) Presumptive synaptic clefts become more extensive and more highly organized. ef $\Lambda x o n$ contacts reconstructed synaptic clefts; primary cleft forms; some secondary clefts remain exposed. $b^{\prime}-e^{\prime}$ represents the hypothetical nerve-dependent de novo sequence, probably at a site other than, but possibly near the original soleplate. $\left.b^{\prime}\right)$ Degeneration of the original muscle fiber, with persistence of basal laminae. $c^{\prime}-d^{\prime}$ ) Regeneration of myotube prior to innervation; no specializations are present. e') Formation of shallow secondary clefts subsequent to contact of the axon with the regenerated myofiber. It is postulated that either the nerve-dependent or the nerve-independent pathway may lead to the formation of a soleplate in mature regenerates that is normal in appearance (f). 
formed de novo in newly innervated embryonic muscle (Hirano, 1967; Teräväinen, 1968; Kelly and Zacks, 1969; Juntenen, 1971; Atsumi, 1977) or ectopically formed soleplates in mature muscle experimentally reinnervated at a new site (Saito and Zacks, 1969; Koening, 1973; Kornielussen and Sommerschild, 1976). The morphogenesis of these nerve-dependent soleplates is depicted schematically in Figure 10. These nerve-dependent soleplates in the EDL grafts were sometimes found within neuromuscular junctions that had other regions that appeared to be reconstructed soleplates. Similar poorly developed soleplates were found even in long-term grafts.

It appeared that the majority of the synapses in the regenerating EDL muscle were made at the reconstructed soleplates. Those soleplates that appeared to have developed de novo subsequent to innervation were frequently located near the reconstructed soleplates. These observations suggest that the nerves are attracted back to the site where the original muscle fiber was innervated. Marshall et al. (1977), Sanes et al. (1978), and Bader (1980) have presented evidence suggesting that nerves in both regenerating mammalian or amphibian muscle are preferentially attracted to settle down at the site of the original neuromuscular junction. In the present study some of the terminal branches of nerves attracted back to the general region of the original soleplate may have settled at new sites and thereby induced soleplates to form de novo.

Two earlier ultrastructural studies of the morphogenesis of soleplates in regenerating mammalian muscle failed to show any evidence of reconstruction of the soleplate. Rather, de novo formation of soleplates appeared to be induced by axonal contact. Bennett et al. (1974) used the grafted EDL from the rat, but the muscle was minced, rather than left intact. This model generally results in more connective tissue within the graft. The connective tissue may have prevented nerves from returning to the sites of the original soleplates. The nerves would then have settled at different sites and induced soleplates to form de novo. Soleplates may have been reconstructed at the original sites but were not detected in the samples used for electron microscopy. In a study by Jirmanova (1975), muscle degeneration was induced in mouse lumbrical muscle by intrasmuscular injection of Marcaine. Unlike the present model, in which Marcaine was used in combination with grafting, nerves did not degenerate in Jirmanova's model. Thus, innervation of myotubes was possible at a very early stage of regeneration before PSCs might have developed. Jirmanova's experimental model is probably closer to the chronological sequence of nerve-muscle interaction during embryogenesis than is the model used in the present study.

The present study and others cited suggest that the regenerated muscle differs significantly from embryonic muscle. Whether or not the regenerated muscle ultimately becomes innervated, it apparently has morphogenetic information that is not expressed in the embryo prior to innervation. This reconstruction of soleplates at or near the original site and the apparent reinnervation of the original site suggests that morphogenetic factors persist in the regenerating muscle. Potential sources of morphogenetic information include Schwann cells or the basal laminae surrounding the original muscle fibers or Schwann cells since these are among the few elements that persist following the degeneration of the original muscle fibers. Burden et al. (1979) and Bader (1981) have postulated that persisting basal laminae or Schwann cells may be capable of inducing the appearance of acetylcholine receptors at these sites. Marshall et al. (1977) and Sanes et al. (1978) have shown that in amphibians nerves will return to the original basal laminae persisting at the original site even if regeneration of the muscle fibers is prevented by irradiation.

\section{ACKNOWLEDGMENTS}

I am indebted to Dr. Bruce Carlson for his helpful suggestions while the work was in progress and during the preparation of the manuscript. I wish to express my appreciation to Jill Rheinheimer and John Beckerman for preparation of the micrographs and photographs, Dennis Goebbel for preparing the illustrations, and Loretta Santana for typing this manuscript.

This study was supported by a grant from the Muscular Dystrophy Association of America, an NIH grant (No. NS 13116) to Dr. Bruce Carlson, and USPH grant No. NS-17017.

\section{LITERATURE CITED}

Albrook, D.B., and J.T. Aitken (1951) Reinnervation of striated muscle after acute ischaemia. J. Anat., 85:376388. 
Atsumi, S. (1977) Development of neuromuscular junctions of fast and slow muscles in the chick embryo: A light and electron microscopic study. J. Neurocytol., 6:691-709.

Bader, D. (1980) Reinnervation of motor endplate-containing and motor endplate-less muscle grafts. Dev. Biol., 77:315-327.

Bader, D. (1981) Density and distribution of $\alpha$-bungarotoxin-binding sites in postsynaptic structures of regenerated rat skeletal muscle. J. Cell Biol., 88:338-345.

Bennett, M.R., T. Florin, and R. Woog (1974) The formation of synapses in regenerating mammalian striated muscle. J. Physiol., 238:79-92.

Burden, S.J., P.G. Sargent, and U.J. McMahan (1979) Acetylcholine receptors in regenerating muscle accumulate at original synaptic sites in the absence of nerve. f. Cell Biol., 82:412-425.

Carlson, B.M. (1973) The regeneration of skeletal muscle-A review. Am. J. Anat., 137:119-150.

Carlson, B.M. (1978) A review of muscle transplantation in mammals. J. Physiol. Bohemoslav., 27:387-400.

Carlson, B.M., and E. Gutmann (1976) Free grafting of the extensor digitorum longus muscle in the rat after marcaine pretreatment. J. Exp. Neurol., 53:82-93.

Carlson, B.M., F.M. Hansen-Smith, and D.K. Magon (1979a) The life history of a free muscle graft. In Muscle Regeneration. A. Mauro, ed. Raven Press, N.Y., pp. 493-507.

Carlson, B.M., K.R. Wagner, and S.R. Max (1979b) Reinnervation of rat extensor digitorum longus muscles after free grafting. Muscle Nerve, 2:304-307.

Clark, W.E., LeGros, and H.S. Wajda (1947) The growth and maturation of regenerating striated muscle fibers. J. Anat., 81:56-63.

Fertuck, C., and M. Salpeter (1974) Localization of acetylcholine receptor by ${ }^{125}$ T-labeled -bungarotoxin binding at mouse motor end plates. Proc. Natl. Acad. Sci. USA, $71: 1376-1378$.

Goshgarian, H.G. (1977) Research Note: A rapid silver impregnation for central and peripheral nerve fibers in paraffin and frozen sections. J. Exp. Neurol., 57:296301 .

Hansen-Smith, F.M., and B.M. Carlson (1979) Cellular responses to free grafting of the extensor digitorum longus muscle of the rat. J. Neurol. Sci., 41:149-173.

Hirano, H. (1967) Ultrastructural study on the morphogenesis of the neuromuscular function in the skeletal muscle of the chick. Z. Zellforsch, 70:198-208.

Jirmanova, I. (1975) Ultrastructure of motor endplates during pharmacologically induced degeneration and subsequent regeneration of skeletal muscle. J. Neurocytol., 4:141-155

Juntunen, J. (1979) Morphogenesis of the cholinergic synapse in striated muscle. Prog. Brain Res., 49:351358.

Karnovsky, M.J. (1964) The localization of cholinesterase activity in rat cardiac muscle by electron microscopy. J. Cell Biol., 23:217-232.

Kelly, A.M., and S.I Zacks (1969) The fine structure of motor endplate morphogenesis. J. Cell Biol., 42:154169.

Koenig, J. (1973) Morphogenesis of motor endplates in vivo and in vitro. J. Brain Res., 62:361-365.

Korneliussen, H., and H. Sommerschild (1976) Ultrastructure of the new neuromuscular junctions formed during reinnervation of rat soleus muscle by a "foreign" nerve. Cell Tissue Res., 167:439-452.

Lentz, T.L. (1969) Development of the neuromuscular junction. J. Cell Biol., 42:431-443.

Marshall, L.M., J.R. Sanes, and U.J. McMahan (1977) Reinnervation of original synaptic sites on muscle fiber basement membrane after disruption of the muscle cells. Proc. Natl. Acad. Sci. USA, 74:3073-3077.

Ogata, T, and F. Murata (1969) Fine structure of motor endplate in red, white, and intermediate fibers of mammalian fast muscle. Tohoku J. Exp. Med., 98:107115.

Padykula, H., and G. Gauthier (1970) The ultrastructure of the neuromuscular junction of mammalian red, white, and intermediate skeletal muscle fibers. $J$. Cell Biol., 46:27-41.

Saito, A., and S.I. Zacks (1969) Fine structure of neuromuscular junctions after nerve section and implantation of nerve in denervated muscle. Exp. Mol. Pathol., 10:256-273.

Sanes, J.R., L.M. Marshall, and U.J. MeMahan (1978) Reinnervation of muscle fiber basal lamina after removal of myofibers. J. Cell Biol., 78:176-198.

Studitsky, A.N. (1963) Dynamics of the Development of Myogenic Tissue Under Conditions of Explantation and Transplantation Cinemicrography in Cell Biology. Acad. Press., N.Y.

Teräväinen, H. (1968) Development of the myoneural junction in the rat. Z. Zellforsch., 87:249-265. 